\title{
BRINGING MEMS TO MARKET
}

\author{
Kurt E. Petersen \\ Cepheid \\ Sunnyvale, CA 94089
}

\section{ABSTRACT}

MEMS innovations are revolutionizing a number of existing and emerging high technology engineering disciplines. However, many problems and delays have been encountered in the intro-duction of MEMS innovations and potential products into commercial market-places. We present here some practical guidelines to evaluate product marketability and to facilitate the transition of a MEMS concept to commercial viability. These guidelines have been gleaned from an analysis of many successful, as well as some unsuccessful, commercialization efforts, a few of which are briefly described here.

\section{INTRODUCTION}

As of the year 2000, we have completed over 25 years of MEMS research, and introduced numerous MEMS products. The field has passed through several phases;

- largely unknown for much of the 70's

- recognized as having a lot of potential in the $80^{\prime} \mathrm{s}$

- having many commercial success stories in the 90's

- now $\rightarrow$ becoming a commonly employed and wellaccepted solution over a wide range of today's technical industries

Few high tech fields have not been influenced by MEMS; if not through the direct introduction of MEMS products, then through compelling R\&D activities and developments which challenge and defy virtually every traditional/conventional mech-anical, opto-mechanical, electromechanical, chemical, and fluidic engineering discipline. The Boeing 747 has 6 million mechanical parts, about 50,000 moving parts. TI's micromirror display chip has 1 million moving mechanical parts. Analog Devices' accelero-meter chip detects and accurately controls motions of on the order of nanometers. ACLARA and Caliper microfluidic chips control fluid volumes in the range of picoliters. MEMS has fundamentally enhanced and vastly expanded the limits of the modern practice of engineering.

However, the very revolutionary nature of MEMS has created real difficulties in the transfer of MEMS innovations to commercial products. Most of these problems have been extensively discussed by many workers in the field at many Hilton Head rump sessions: the unique complications of MEMS packaging, the question to integrate or not to integrate, the problem of MEMS foundries, the unique difficulties of MEMS testing, the unique processing techniques of MEMS, the inter-disciplinary nature of MEMS, the comparison of MEMS chips to integrated circuit chips. In the same way that integrated circuit technology has fundamentally redefined the practice of electrical engineering, MEMS is fundamentally redefining many other engineering disciplines. Such momentous changes do not come easily or quickly.

\section{INTRODUCING MEMS PRODUCTS}

Introducing a new, revolutionary tcchnology across a variety of markets requires us to carefully consider the commercial accep-tance of that technology from several viewpoints.

One succinct criteria for successfully introducing, manufacturing, and marketing a MEMS product is to ensure that the MEMS device brings a "compelling, enabling, and rational technology into a starved market segment".

This means that the device must provide a compelling function which enables a major performance advantage over existing products, must have a very robust manufacturing process, and must be readily adopted into a very receptive marketplace which is anxiously searching for a solution to the problem which the device addresses.

During the past 25 years of MEMS, this formula for success has been repeated again and again. There have also been examples which did not (or could not) follow this formula, and failed. One frustrating characteristic of the formula is that, many times, a compelling, enabling, rational technology has been forced to wait many years for that very crucial starving market segment to develop. Without the starving market, however, the application will either fail, will be only marginally successful, or will be forced to wait longer for the market to develop.

Another factor which greatly influences time to market for an enabling device concept can be characterized as a necessary "convergence of technologies". For example, surface micromachined optical modulators were first demonstrated almost 30 years ago. However, in order to make use of such an optical element in a practical projection display product, a low-cost manufacturing infrastructure must also be in place for fabricating at least $500 \mathrm{~K}$ optical modulators on a single chip with near perfect yield. Such a large number of elements is required to achieve a practical display. No manufacturing technology with this capa-bility existed for any type of chip until the late 80's (see Figure 1). The convergence of optical modulator array technology and fabrication technologies for producing large numbers of elements on a single chip converged in the early 90's, making it possible to launch products based on TI's micromirror technology.

Another important consideration is that product introduction plans which treat MEMS devices as simple components in larger systems are a mistake. It takes an enormous investment in effort and time to design, develop, optimize, and move into production a MEMS chip. In many instances, the complete product system incorporating the MEMS chip is designed, developed, optimized, and moved into production only after the performance character-istics of the chip are finalized. The sequential nature of such product devclopment effort can postpone actual sales for many years. Whenever possible, the MEMS developer/manufacturer should strive to develop the entire product in a parallel fashion, thereby dramatically reducing time to market. 


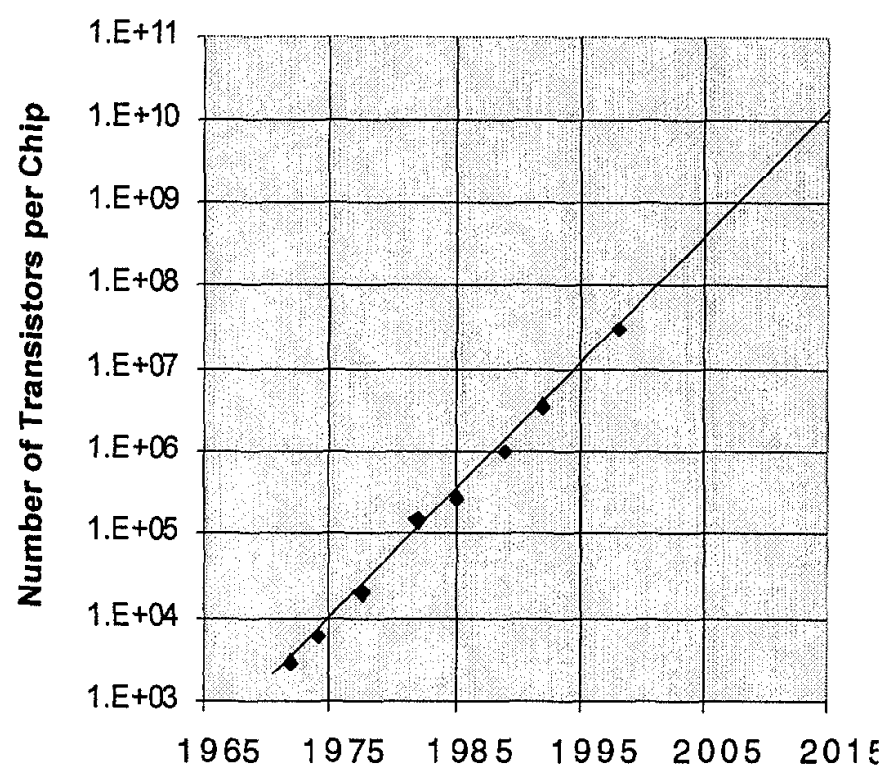

Figure 1. The number of electronic devices per microprocessor chip has followed "Moore's Law" for 30 years. Even though micromechanical optical display elements had been in existence since the 70 's, it was not until after about 1990 that the capability of manufacturing chips with sufficient optical elements (about 1 million) to create a viable display product became possible and practical for the first time.

IC-like batch fabrication of MEMS devices is often touted as a major advantage over "one-at-a-time" fabrication. This is a mis-conception for many mechanical devices. Very rapid, fully-automated, one-at-a-time manufacturing can be extremely cost-effective, easily producing $10^{\prime} \mathrm{s}$ of millions of relatively complex parts per year for capital investments of less than $\$ 1 \mathrm{M}$. It can be a mistake to lightly dismiss such simple, wellestablished manu-facturing processes and devices as serious competition to batch-produced MEMS chips.

The implication that market pull dominates successful MEMS products is, of course, only partly true. Market pull is very influ-ential and its importance cannot be under estimated. However, there is no "market pull" without the fundamental progress, promise, and inspiration of advanced technological innovations. Basic research and advanced technical demonstrations always set the stage and establish the expectations for future commercial products.

\section{SUCCESS STORIES (AND FAILURES)}

\section{Pressure Sensors}

Until about 1982, MEMS pressure sensors were employed only in very limited, high-end, expensive aerospace and industrial applications. During the early $80^{\prime} \mathrm{s}$, new federal regulations man-dated reduced fuel emissions for automobiles. The solution found by the automotive industry required a pressure sensor to help con-trol the fuel/air ratio. Suddenly, there was a huge demand for low-cost, electronic-based, reliable pressure sensors; a starving market was born and MEMS pressure sensors easily dominated the market. Furthermore, MEMS dominance in this important market was readily transferred to other large markets including disposable medical blood pressure sensors and consumer applications.

\section{Accelerometers}

The first MEMS acceleration sensors (demonstrated at Stanford and at Westinghouse in the 70's) languished on the shelf for nearly 20 years. As American quality of life continued to grow during the $70^{\prime} \mathrm{s}$ and $80^{\prime} \mathrm{s}$, however, automobile safety became a dominate political issue. The furious introduction of air-bag crash-protection systems in the early 90's created a starving market for low-cost, high reliability, electronic-based crash sensors accelero-meters). These MEMS devices now completely dominate the air-bag market and are spinning off into other areas such as low-cost gyroscopes, navigation cquipment, and virtual reality sensors.

\section{Projection Displays}

Micromechanical optical modulators have a renowned history going back to the early 70's. Texas Instruments began working on large two-dimensional modulator arrays in the late 70 's. This technology barely survived 2 decades of corporate politics, an unimpressed marketplace, and the complete dominance of CRT and liquid crystal displays. The proliferation of computers, however, has generated a starving market for high performance, digitally-compatible displays in the late 80's which continues today. The recent success of TI's micromirror display was also helped by a convergence of technologies. Before about 1990, it was impossible to even consider the idea of fabricating cost-effec-tive display chips with the required millions of active elements. Advances in IC fabrication technology, exemplified by the 64 megabyte momory chip, has changed all that. In the early 90's, the convergence of MEMS optical modulation technology with manu-facturing techniques capable of creating millions of elements on a single chip suddenly made the micromirror display commercially feasible.

\section{Ink Jet Nozzles}

First developed at IBM in the 70's, it took about 20 years for micromachined ink jet nozzles to become nearly commodity items. Today, over 100M ink jet chips are produced every year. Despite the belief that we have been supposedly moving toward the "paperless" world for 30 years, the market is still starving for simple, low-cost printers with more and more demand for high quality. In fact, the only practical technology for achieving the demands of low-cost, photographic-quality, color printers for today's graphicsoriented computers, continues to ink jet printing.

\section{Chemical Sensors}

Throughout many years of development during the 70's and 80's, ISFETs and CHEMFETs suffered from serious performance problems caused by drift and instability. Finally, companies such as i-STAT recognized a starving market for chemical sensors and took practical advantage these unstable devices. Instead of trying to solve the intractable problems with drift and instability, i-STAT created a business in which the MEMS sensors were very low-cost, used only once, then disposed of. While the i-STAT technology is a model for efficient and elegant integration of MEMS and plastic packaging, it took i-STAT over ten years to convince medical practitioners that the market was starving for fast, disposable blood chemistry sensors. There are still many other market segments for such devices and increased developments in microfluidic technol-ogy will allow further integration and greater demand for chemical analysis and chemical sensors. Since many of the applications for this technology are medical and biomedical, the move toward dis-posable sensors and microfluidic systems will grow dramatically. 
Fiber Optic Switches

Since the dawn of MEMS, numerous workers in the field (the author included) have continuously predicted that this technology would become important for optical fiber switching devices. Suddenly, within the last year, this application area has exploded with intense activity. Why? The basic MEMS technologies now being applied to fiber switches have been around for over 10 years. What has changed is the market. With the advent of the internet, cable television, and video-ondemand, the requirement for communication bandwidth has bccome insatiable. Wc arc now cxpcriencing a starving market for any technical innovations which will enable the implementation and rapid expansion of fiber optic communication systems.

\section{Micro-valves}

At the other end of the spectrum for MEMS success stories is the elusive micro-valve application and product. Numerous types and versions of MEMS micro-valves have been demonstrated, developed, and introduced into the market during the past 15 years or longer. None of these have become notable successes because the market is not starving for such products; numerous low-cost traditional valves are readily available which perform perfectly adequately for every conceivable application today. Are there any compelling technical reasons why a MEMS valve should be preferred over a conventional valve? Of course, there are excep-tions and these exceptions can guide us to future successful valves. The Microsensor gas chromatograph system, for example, employs an array of micro-valves in a system where low dead-volume is a crucial performance parameter. In this case, the accomplishment of a high density, integrated array of valves provides the unique operating advantage required in this application. MEMS micro-valves can provide extremely important cost and performance advantages when used in arrays. In the future, in market applica-tions which require highly integrated fluidic systems, the specific advantages of MEMS valves will become valuable. As of today, however, MEMS micro-valves, independent of such highly integrated fluidic systems, have not yet become commercially successful.

\section{Microelectromechanical Switches}

The first microelectromechanical switches were fabricated at Westinghouse and IBM in the 70's. Since then, numerous un-successful efforts have been undertaken to commercialize these devices. The lack of success to date has been the result of two problems. Most importantly, there is not a starved market for electromechanical switches. Today, these devices are basically commodities. Numerous, high-reliability, low-cost devices are readily available from many suppliers. (Most of these are manufactured with highly automated on-at-a-time assembly lines.) MEMS switches do not provide a compelling incentive for replacing conventional relays in traditional applications for relays. At the same time, the most common applications for conventional relays are for the switching of relatively high DC currents - on the order of 1 ampere or more. It has been extremely difficult to design and demonstrate MEMS switches capable of reliably controlling such highcurrent $\mathrm{DC}$ levels. In recent years, however, there has been a trend to design MEMS switches specifically for RF applications. This new application area may allow the MEMS switch, invented over 20 years ago, finally successful. In addition, new applications for arrays of electromechanical switches may provide the basis for successful MEMS switch products in the future.

These examples do not mean that every application is driven solely by market pull and that research in MEMS does not also provide a high level of technology push. Indeed, no product would ever be possible if the early research and feasibility phases were not conceived, initiated, and successfully completed. However, no product will ever become commercially successful without

- the proven, compelling concept

- the enabling device performance capability

- the rational manufacturing process established

- and the starving market application

\section{THE FUTURE}

So, can we look into the future and anticipate some of the MEMS success stories over the next 10 years? One interesting exercise we can do is extrapolate what has been happening over the past 10-20 years with computing power and data recording density, two powerful manufacturing disciplines which drive advances in modern technology. From Figures 1 and 2, we can expect factors of improvement in these areas of between 100 and 1,000 over the next 15 years. Memory chips can be expected to hold a quantity of data equivalent to yesterday's hard disk drives. If current trends continue, transistors will average less than $0.2 \mu \mathrm{m}$ on a side. If current trends continue, hard disk drives (currently at about $15 \mathrm{bits} / \mu^{2}$ ) will approach $5 \times 10^{13}$ bits $/ \mathrm{in}^{2}$, or $100 \mathrm{~K}$ bits $/ \mu \mathrm{m}^{2}$, or $10 \mathrm{~nm}^{2} / \mathrm{bit}$ - that's $3 \mathrm{~nm}$ or $30 \AA$ on a side) ! This implies that two million elements (electronic OR mechanical) photolithographically produced on a single chip, will be trivial. On the other hand, accurate location and tracking of individual bits of data will require extremely high precision mechanical devices. All of these trends indicate an increased requirement for MEMS devices (such as micromechanical actuators) as well as an enhanced ease of manufacture of micromechanical arrays (such as MEMS displays, or actuator arrays). The future of MEMS is promising in many ways and will continue to grow along with the expected progress in photolithographic fabrication technology.

\section{Microfluidics Related to Biomedical Applications}

A significant revolution is currently underway in the develop-ment and application of microfluidic techniques to a variety of medical, biomedical, and chemical processing and delivery requirements. In a sense, the ink jet nozzle was the initial predecessor of this new microfluidic field. However, microfluidics is only a single area in a much broader and more influential movement in which integrated circuit based technologies are being increasingly applied to biomedical and chemical applications. The Affymetrix Gene chip and other DNA hybridization arrays are a part of this effort. This trend will continue to have an enormous impact on the future of biomedical and biochemical development as well as every other possible area of bioanalysis and diagnostics. Not only will MEMS technologies be employed in pharmaceutical development and genomic discovery, but also in the practical implementation of this knowledge on a daily basis for individual paticnts in a hospital or a doctor's officc. For example, future therapeutic drugs will only be prescribed to patients who have been genetically tested to ensure that they have the proper genetic profile to respond efficaciously to the drug. This new area is called pharmacogenomics. In a few years, it will be common to have your blood genetically analyzed in your doctor's office, by MEMS devices, within just a few minutes, to determine your 
genetic compatibility with a variety of drugs, allowing the physician to appropriately prescribe the correct drug for you. These markets are starving for fast, miniaturized, automated techniques for performing these and other bioanalytical functions.

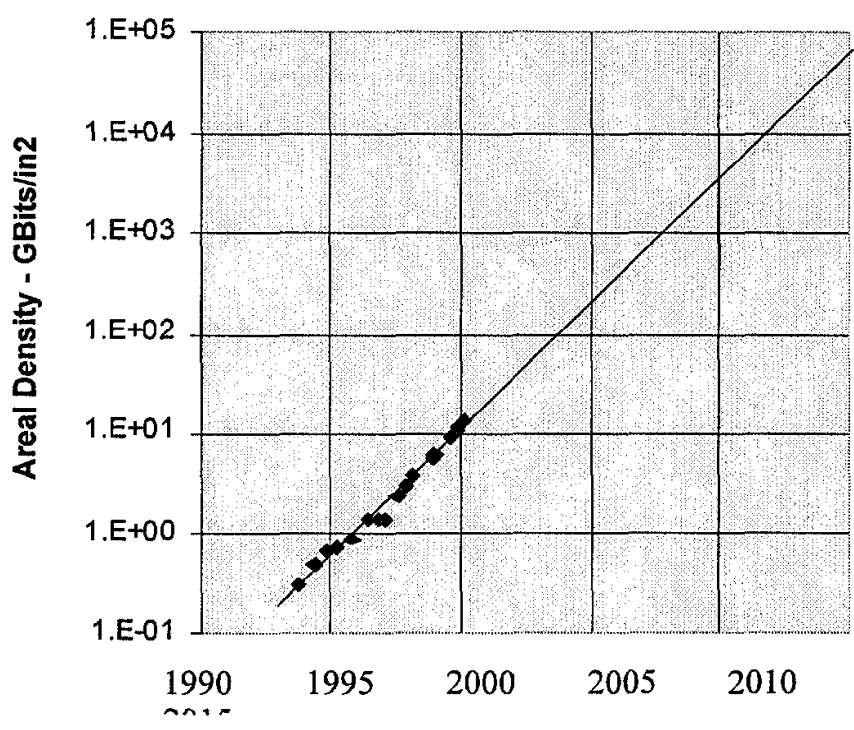

Figure 2. The areal density of magnetic recording products continues to increase exponentially in the same way as the number of transistors/chip, predicted by Moore's Law.

\section{Data Storage}

Continued expansion of the internet and demand for video is driving storage technology. As fiber optic and other high band-width capability becomes increasingly common, demand for storage will continue to grow exponentially for many years. This market is always starving for higher data density and lower cost. Because the current technical approaches have not yet reached their technical limits, MEMS has yet to make a commercial impact on this ubiquitous technology - no starving market, yet. However, it is virtually inevitable that future data storage devices will employ MEMS devices for various purposes: micro-positioning and tracking devices for accurately accessing sub-micron magnetic, optical, or surface profile (AFM) data tracks; micro-mirrors for scanning and positioning of optical beams; micro-beams for scanning and controlling Atomic Force Microscope (AFM) recording and reading tips.

\section{Microsurgical Instruments}

Many advances in surgery are guided by the trend toward non-invasive techniques, intravascular devices, and laprascopic procedures. It may be possible for MEMS to contribule to this important and growing technical direction. Microsensors, scan-ners, surgical devices, and implantable devices are all biomedical candidates for MEMS development.

\section{$R F$ Communications}

As a essential feature of the movement toward absolute portability, wireless communication has many opportunities for improvements, enhancements, increased electronic integration, and cost reductions. One recent start-up has begun to design dispos-able cell-phones - like disposable cameras. Although much work and many innovations are still needed, resonant MEMS structures have the potential to replace conventional electronic filters and tunable frequency standards. Tunable mechanical filters, integ-rated with high frequency electronics could achieve the ultimate goal of a single RF transceiver chip. MEMS electronic switches, conceived and demonstrated almost 25 years ago, are already close to commercial implementation in RF applications. For many years, the development of these electromechanical devices were targeted toward traditional DC switching applications. However, DC switches are very low-cost commodities without a starving market. Current RF switches, on the other hand, are expensive components with sub-optimal performance, which are increasingly in demand for the starving RF communications market.

\section{Energy Storage}

All the full-function capabilities of future computation and communication must be completely portable. The primary limit-ation to this portability is energy storage. Today's batteries are simply inadequate and will become even more problematic. In addition, the long-anticipated mobile robotic machines of the future will not be possible without quantum improvements in mobile power generation and storage. Orders of magnitude increases in energy storage will revolutionize technology. Can MEMS contribute to such a revolution? Aggressive and forward-looking projects such as the MIT microturbine device have the potential to create power sources more than 10 times more efficient in weight and size than the best batteries. However, other (possibly simpler) approaches using integrated gas and liquid fuel-flow systems together with micro-electric generators might be possible. Energy storage is a market which is always starving for improvement.

\section{Displays}

Cell phones, PDAs, and other hand held electronic devices have terrible displays today. Even the impressive displays on lap-top computers are power hungry and perform very poorly in sunlight. Promising micro-displays which fit into glasses or goggles have been developed and are close to commercialization. The demand for enhanced performance in every display market creates a demanding, constantly starving market for new and improved approaches. MEMS will continue to contribute to all these display applications, micro-displays, projection displays, as well as for new, effective direct view displays.

\section{ECONOMIC CONSIDERATIONS}

In bringing new MEMS developments and products to market, it is crucial to understand the economic implications of our guiding criteria - "applying a compelling, enabling, and rational technology into a starved market segment". The first obvious economic axiom is that we must be able to manufacture the device and sell it at a cost which is consistent with market requirements. Lets look at an example. Suppose we have a product concept which we anticipate can be totally developed and moved to production for \$10M (including the cost of the manufacturing equipment and facility). This is not an unreasonable amount -15 or 20 people working for 2 years. Simplistically, in order to make a profit on this product and grow the company, the part must be sold for a price which includes the materials and manufacturing costs, plus overhead, plus a profit, plus amortization of the $\$ 10 \mathrm{M}$ investment. If sales are only $200 \mathrm{~K}$ parts/year, at least $\$ 10$ in amortization costs must be added to the price of each part. If sales are $2 \mathrm{M}$ parts/year, about $\$ 1$ must be added to the price of each part. The amortization costs should only be about $10-20 \%$ of the price of the part. 


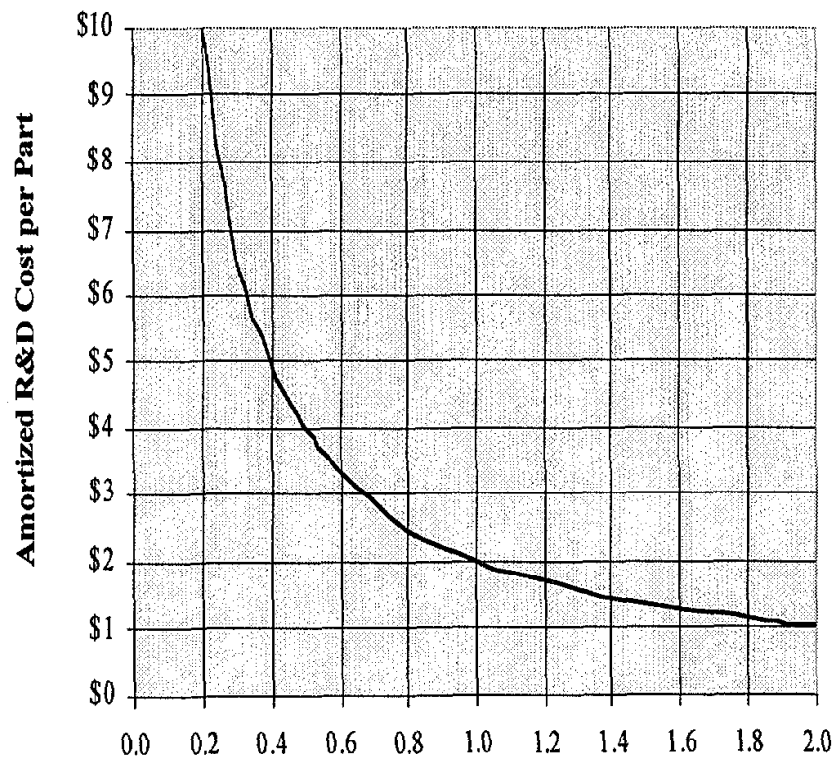

Figure 3. This graph shows the additional amortization cost which should be added to the cost of a product depending on the number of parts sold per year. It assumes that the total costs for product development and production facilities are $\$ 10 M$ and the amortization period is 5 years.

There is an important lesson here. If the required price target for the part is on the order of $\$ 10$, sales quantities must be more than about 1-2M parts/year to justify the development costs. At the same time, the target price must be on the order of $\$ 100$ if the sales quantities are only about $100-200 \mathrm{~K}$ parts/year. For these reasons, low- and medium-cost, commodity-type, replacement products should be avoided.

\section{CONCLUSIONS}

The opportunities for developing and marketing successful products, based on MEMS technologies, are enormous. The infra-structure now in place for MEMS, including university research and education programs, equipment manufacturers, modeling and design software, foundries and services, government $R \& D$ funding, conference and journals, acceptance by venture capital funding groups, all provide a technical and informational foundation which is invaluable to MEMS entrepreneurs. This large base of support, together with the current high degree of commercial success, acceptance, and credibility of MEMS is making it easier to bring important new MEMS products to the market.

However, the past 25 year history of MEMS products suggest some current guidelines which will improve the prospects for the successful introduction of new products based on MEMS. As the field changes and progresses, the guidelines will change. As research continues, innovative new devices and ncw applications will be conceived. It is an exciting time for a potent and dynamic technology. 